\title{
OPTIMAL TIKHONOV APPROXIMATION FOR A SIDEWAYS PARABOLIC EQUATION
}

\author{
CHU-LI FU, HONG-FANG LI, XIANG-TUAN XIONG, AND PENG FU
}

Received 27 April 2004 and in revised form 31 December 2004

We consider an inverse heat conduction problem with convection term which appears in some applied subjects. This problem is ill posed in the sense that the solution (if it exists) does not depend continuously on the data. A generalized Tikhonov regularization method for this problem is given, which realizes the best possible accuracy.

\section{Introduction}

In many industrial applications one wants to determine the temperature on the surface of a body, where the surface itself is inaccessible to measurement $[2,4]$. In this case it is necessary to determine surface temperature from a measured temperature history at a fixed location inside the body. This problem is called an inverse heat conduction problem (IHCP). In a one-dimensional setting, assuming that the body is large, the following model problem or the standard sideways heat equation:

$$
\begin{gathered}
u_{t}=u_{x x}, \quad x>0, t>0, \\
u(x, 0)=0, \quad x \geq 0, \\
u(1, t)=g(t), \quad t \geq 0,\left.\quad u(x, t)\right|_{x \rightarrow \infty} \text { bounded }
\end{gathered}
$$

has been discussed by many authors $[4,6,7,10,16,18,19,20]$. But when a fluid is flowing through the solid, for example, a gas is travelling from the rear surface, there must be a convection term in heat conduction equation $[1,17]$. A model problem in this case is the following sideways parabolic equation with nondivergence type in the quarter plane [13]:

$$
\begin{gathered}
u_{t}+u_{x}=u_{x x}, \quad x>0, t>0, \\
u(x, 0)=0, \quad x \geq 0, \\
u(1, t)=g(t), \quad t \geq 0,\left.\quad u(x, t)\right|_{x \rightarrow \infty} \text { bounded. }
\end{gathered}
$$

We want to know the solution $u(x, t)$ for $0 \leq x<1$. This problem is ill posed in the sense that the solution (if it exists) does not depend continuously on the data. Some regularization methods and error estimates have been given in $[12,13,23]$; we have even obtained 
some results for the following more general sideways parabolic equation:

$$
\begin{gathered}
u_{t}=a(x) u_{x x}+b(x) u_{x}+c(x) u, \quad x>0, t>0, \\
u(x, 0)=0, \quad x \geq 0, \\
u(1, t)=g(t), \quad t \geq 0,
\end{gathered}
$$

where $a, b$, and $c$ are known functions which satisfy some specified conditions $[9,11$, 14]. Especially, the uniqueness of solution of problem (1.3) was explained in [14]. The uniqueness of solution of problem (1.2) can also be similarly established according to [3]. But all the error estimates between the regularized approximate solutions based on above methods and exact solutions are at most of optimal order.

There are some papers, for example $[5,15]$, in which the a priori choice of the regularizing parameters is generally given for the "optimal convergence rate" based on the conditional stability. But their optimal convergence rate is only the convergence with optimal order (cf. Definition 2.1(ii)), but not optimal (cf. Definition 2.1(i)). In addition, the conditions of "conditional stability" is stronger than our priori assumption (1.6).

Now our interest is to give a new regularization method for problem (1.2), which will be called generalized Tikhonov regularization method, such that the error estimate of this method possesses the best possible accuracy, that is, the error estimate of this method is optimal. It is well known that it is much more difficult to prove results about optimality instead of just order optimality [8, page 75]. So far, as far as we know, the unique result about optimality of IHCP is obtained only for the standard sideways heat equation (1.1) $[20,21]$.

As we consider the problem $(1.2)$ in $L^{2}(\mathbb{R})$ or $H_{p}(\mathbb{R})$ with respect to the variable $t$, we extend the domain of definition of the functions $u(x, \cdot), g(\cdot):=u(1, \cdot), f(\cdot):=u(0, \cdot)$ and other functions appearing in the paper to the whole real $t$-axis by defining them to be zero for $t<0$. The notation $\|\cdot\|,(\cdot, \cdot)$ denotes $L^{2}$-norm and $L^{2}$-inner product, respectively,

$$
\hat{h}(\xi):=\frac{1}{\sqrt{2 \pi}} \int_{-\infty}^{\infty} e^{-i \xi t} h(t) d t
$$

is the Fourier transform of function $h(t)$, and

$$
\|h\|_{p}:=\left(\int_{-\infty}^{\infty}\left(1+\xi^{2}\right)^{p}|\hat{h}(\xi)|^{2} d \xi\right)^{1 / 2}
$$

is the $H_{p}$-norm of the function $h(t)$. We assume that there exists a priori bound for $f(t):=u(0, t)$ :

$$
\|f\|_{p} \leq E, \quad p \geq 0
$$

Let $g(t)$ and $g_{\delta}(t)$ denote the exact and measured (noisy) data at $x=1$ of solution $u(x, t)$, respectively, which satisfy

$$
\left\|g(\cdot)-g_{\delta}(\cdot)\right\| \leq \delta .
$$


For the uniqueness of solution, we require that $\|u(x, \cdot)\|$ be bounded [14]. The solution of problem (1.2) has been given in $[12,13,17,23]$ by

$$
u(x, t)=\frac{1}{\sqrt{2 \pi}} \int_{-\infty}^{\infty} e^{i \xi t} e^{(1-x) \theta(\xi)} \hat{g}(\xi) d \xi, \quad 0 \leq x<1,
$$

or equivalently,

$$
\widehat{u}(x, \xi)=e^{(1-x) \theta(\xi)} \widehat{g}(\xi), \quad 0 \leq x<1,
$$

where

$$
\begin{gathered}
\theta(\xi)=\sqrt{i \xi+\frac{1}{4}}-\frac{1}{2}=\frac{1}{2}\left[\sqrt[4]{1+16 \xi^{2}}\left(\cos \frac{\beta}{2}+i \sin \frac{\beta}{2}\right)-1\right], \quad \xi \in \mathbb{R}, \\
\beta=\arg (1+4 \xi i), \quad \tan \beta=4 \xi, \quad-\frac{\pi}{2}<\beta<\frac{\pi}{2}, \xi \in \mathbb{R} \\
\cos \frac{\beta}{2}=\frac{\sqrt{\sqrt{1+16 \xi^{2}}+1}}{\sqrt{2} \sqrt[4]{1+16 \xi^{2}}}, \quad \xi \in \mathbb{R} .
\end{gathered}
$$

The following representations will be useful and it is easy to see from (1.9) that

$$
\begin{gathered}
\hat{f}(\xi)=e^{\theta(\xi)} \hat{g}(\xi), \\
\hat{u}(x, \xi)=e^{-x \theta(\xi)} \hat{f}(\xi) .
\end{gathered}
$$

\section{Preliminary}

Most facts here are known $[8,21,22]$. We consider arbitrary ill-posed inverse problems

$$
A x=y,
$$

where $A \in \mathscr{L}(X, Y)$ is a linear injective bounded operator between infinite dimensional Hilbert spaces $X$ and $Y$ with nonclosed range $R(A)$ of $A$. We assume that $y^{\delta} \in Y$ are the available noisy data with $\left\|y-y^{\delta}\right\| \leq \delta$. Any operator $R: Y \rightarrow X$ can be considered as a special method for solving (2.1), the approximate solution to (2.1) is then given by $R y^{\delta}$.

Let $M \subset X$ be a bounded set. We introduce the worst-case error $\Delta(\delta, R)$ for identifying $x$ from $y^{\delta} \in Y$ under the assumption $x \in M$ according to

$$
\Delta(\delta, R)=\sup \left\{\left\|R y^{\delta}-x\right\| \mid x \in M, y^{\delta} \in Y,\left\|A x-y^{\delta}\right\| \leq \delta\right\} .
$$

This worst-case error characterizes the maximal error of the method $R$ if the solution $x$ of the problem (2.1) varies in the set $M$.

Definition 2.1. Parameter-dependent methods $R=R_{\delta}$ are called

(i) optimal on the set $M$ if $\Delta\left(\delta, R_{\delta}\right)=\inf _{R} \Delta(\delta, R)$ where the infimum is taken over all methods $R: Y \rightarrow X$,

(ii) order optimal on the set $M$ if $\Delta\left(\delta, R_{\delta}\right) \leq \operatorname{Cinf}_{R} \Delta(\delta, R)$ with $C \geq 1$ holds. 
Now we review some optimality results if the set $M$ has been given by

$$
M_{\varphi, E}=\left\{x \in X \mid x=\left[\varphi\left(A^{*} A\right)\right]^{1 / 2} v,\|v\| \leq E\right\}
$$

or equivalently,

$$
M_{\varphi, E}=\left\{x \in X \mid\left\|\left[\varphi\left(A^{*} A\right)\right]^{-1 / 2} x\right\| \leq E\right\}
$$

where the operator function $\varphi\left(A^{*} A\right)$ is well-defined via the spectral representation

$$
\begin{aligned}
\varphi\left(A^{*} A\right) & =\int_{0}^{a} \varphi(\lambda) d E_{\lambda}, \\
A^{*} A & =\int_{0}^{a} \lambda d E_{\lambda},
\end{aligned}
$$

is the spectral decomposition of $A^{*} A,\left\{E_{\lambda}\right\}$ denotes the spectral family of operator $A^{*} A$, and $a$ is a constant such that $\left\|A^{*} A\right\| \leq a$. In the case when $A: L^{2}(\mathbb{R}) \rightarrow L^{2}(\mathbb{R})$ is a multiplication operator, $A x(s)=a(s) x(s)$, the operator function $\varphi\left(A^{*} A\right)$ takes the form

$$
\varphi\left(A^{*} A\right) x(s)=\varphi\left(|a(s)|^{2}\right) x(s)
$$

A generalized Tikhonov regularized approximation $x_{\alpha}^{\delta}$ is determined by solving the minimization problem

$$
\min _{x \in X} J_{\alpha}(x), \quad J_{\alpha}(x)=\left\|A x-y^{\delta}\right\|^{2}+\alpha\left\|\left[\varphi\left(A^{*} A\right)\right]^{-1 / 2} x\right\|^{2}
$$

or equivalently, by solving the Euler equation

$$
\left(A^{*} A+\alpha\left[\varphi\left(A^{*} A\right)\right]^{-1}\right) x_{\alpha}^{\delta}=A^{*} y^{\delta} .
$$

When $\varphi\left(A^{*} A\right)=I,(2.7)$ and (2.8) are identical with classical Tikhonov regularization.

In order to obtain optimality result, we assume as in [22] that the function $\varphi$ in (2.3) and (2.4) satisfies the following assumption.

Assumption 2.2. The function $\varphi(\lambda):(0, a] \rightarrow(0, \infty)$ (where $a$ is a constant such that $\left.\left\|A^{*} A\right\| \leq a\right)$ is continuous and satisfies the following properties:

(i) $\lim _{\lambda \rightarrow 0} \varphi(\lambda)=0$;

(ii) $\varphi(\lambda)$ is strictly monotonically increasing on $(0, a]$;

(iii) $\rho(\lambda):=\lambda \varphi^{-1}(\lambda):(0, \varphi(a)] \rightarrow(0, a \varphi(a)]$ is convex.

The next theorem gives us a formula for the best possible worst-case error $\inf _{R} \Delta(\delta, R)$, which can be found also in [22]. 
Theorem 2.3. Let $M_{\varphi, E}$ be given by (2.3), let Assumption 2.2 be satisfied, and let $\delta^{2} / E^{2} \in$ $\sigma\left(A^{*} A \varphi\left(A^{*} A\right)\right)$ where $\sigma\left(A^{*} A\right)$ denotes the spectrum of the operator $A^{*} A$, then

$$
\inf _{R} \Delta(\delta, R)=E \sqrt{\rho^{-1}\left(\frac{\delta^{2}}{E^{2}}\right)}
$$

The general Tikhonov regularization method appears to be optimal on the set $M_{\varphi, E}$ given by (2.3) provided the regularization parameter $\alpha$ is chosen properly. For this method there holds [22, Theorem 5.1] the following.

Theorem 2.4. Let $M_{\varphi, E}$ be given by (2.3), let Assumption 2.2 be satisfied, let $\varphi(\lambda):[0, a] \rightarrow$ $\mathbb{R}$ be two times differentiable, let $\rho(\lambda)$ be strictly convex on $(0, \varphi(a)]$, and let $\delta^{2} / E^{2} \leq a \varphi(a)$. If the regularization parameter $\alpha$ is chosen optimally by

$$
\alpha=\frac{\lambda_{0}}{\varphi^{-1}\left(\lambda_{0}\right) \varphi^{\prime}\left(\varphi^{-1}\left(\lambda_{0}\right)\right)}\left(\frac{\delta}{E}\right)^{2} \text { with } \lambda_{0}=\rho^{-1}\left(\frac{\delta^{2}}{E^{2}}\right),
$$

then for the general Tikhonov regularized solution $x_{\alpha}^{\delta}=R_{\alpha} y^{\delta}$ defined by (2.8), there holds the optimal error estimate

$$
\Delta\left(\delta, R_{\alpha}\right) \leq E \sqrt{\rho^{-1}\left(\frac{\delta^{2}}{E^{2}}\right)}
$$

\section{Optimal Tikhonov approximation for the problem (1.2)}

In this section, we consider the generalized Tikhonov regularization method (2.8) for problem (1.2), and based on Theorems 2.3 and 2.4 show how to choose the regularization parameter such that it is optimal.

We introduce the Sobolev scale $\left(H_{p}\right), p \in \mathbb{R}^{+}$of positive real order $p$ according to $H_{0}=L^{2}(\mathbb{R}), H_{p}=\left\{v(t) \in L^{2}(\mathbb{R}) \mid\|v\|_{p}<\infty\right\}$ where $\|v\|_{p}$ is given by (1.5). For problem (1.2) we require a priori smoothness condition concerning the unknown solution $u(x, t)$ according to

$$
u(x, t) \in M_{p, E}:=\left\{u(x, \cdot) \in L^{2}(\mathbb{R}) \mid\|u(0, \cdot)\|_{p}=\|f(\cdot)\|_{p} \leq E \text { for some } p \geq 0\right\} .
$$

Denote the best possible worst-case error as

$$
\omega(\delta, x):=\inf _{R(x)} \Delta(\delta, R(x))
$$

where $R(x): g_{\delta}(\cdot) \rightarrow u^{\delta}(x, \cdot)$ and

$$
\Delta(\delta, R(x))=\sup \left\|R(x) u^{\delta}(1, \cdot)-u(x, \cdot)\right\|=\sup \left\|R(x) g_{\delta}(\cdot)-u(x, \cdot)\right\|
$$

with $g_{\delta}(t)=u^{\delta}(1, t)$ and $g(t)=u(1, t)$ satisfy (1.7), and the supremum is taken over $u(x, t) \in M_{p, E}$ given by (3.1). 
Firstly, we formulate the problem (1.2) of identifying $u(x, t)$ from (unperturbed) data $u(1, t)=g(t)$ as an operator equation

$$
A(x) u(x, t)=g(t), \quad 0 \leq x<1
$$

with a linear operator $A(x) \in \mathscr{L}\left(L^{2}(\mathbb{R}), L^{2}(\mathbb{R})\right)$.

It is easy to know that (3.4) is equivalent to the following operator equation [12]:

$$
\widehat{A}(x) \widehat{u}(x, \xi)=\widehat{g}(\xi) \quad \text { with } \widehat{A}(x)=\mathscr{F} A(x) \mathscr{F}^{-1} \text {, }
$$

where $\mathscr{F}: L^{2}(\mathbb{R}) \rightarrow L^{2}(\mathbb{R})$ is the (unitary) Fourier operator that maps any $L^{2}(\mathbb{R})$ function $h(t)$ into its Fourier transform $\hat{h}(\xi)$ given by (1.4), and we know from (1.9) that

$$
\widehat{A}(x)=e^{-\theta(\xi)(1-x)}=e^{-(1 / 2)\left[\sqrt[4]{1+16 \xi^{2}}(\cos (\beta / 2)+i \sin (\beta / 2))-1\right](1-x)}
$$

where $\hat{A}(x): L^{2}(\mathbb{R}) \rightarrow L^{2}(\mathbb{R})$ is a linear normal operator (multiplication operator) and

$$
\widehat{A}^{*}(x)=e^{-\overline{\theta(\xi)}(1-x)}=e^{-(1 / 2)\left[\sqrt[4]{1+16 \xi^{2}}(\cos (\beta / 2)-i \sin (\beta / 2))-1\right](1-x)},
$$

which is the conjugated operator of $\widehat{A}(x)$, so

$$
\widehat{A}^{*}(x) \widehat{A}(x)=e^{-(\theta(\xi)+\overline{\theta(\xi)})(1-x)}=e^{-\left[\sqrt[4]{1+16 \xi^{2}} \cos (\beta / 2)-1\right](1-x)} .
$$

The a priori smoothness condition (3.1) and the general source set (2.3) can be transformed into their equivalent condition in the frequency domain as follows:

$$
\begin{aligned}
\widehat{u}(x, \xi) \in \widehat{M}_{p, E}=\left\{\widehat{u}(x, \xi) \in L^{2}(\mathbb{R}) \mid\left\|\left(1+\xi^{2}\right)^{p / 2} \widehat{u}(0, \xi)\right\|\right. \\
\left.=\left\|\left(1+\xi^{2}\right)^{p / 2} \widehat{f}(\xi)\right\| \leq E, \text { for some } p \geq 0\right\} \\
\widehat{M}_{\varphi, E}=\left\{\hat{u}(x, \cdot) \in L^{2}(\mathbb{R}) \mid\left\|\left[\varphi\left(\widehat{A}^{*}(x) \hat{A}(x)\right)\right]^{-1 / 2} \widehat{u}(x, \cdot)\right\| \leq E\right\} .
\end{aligned}
$$

By their equivalence, it is easy to know that the representation of $\varphi(\lambda)$ is given (in parameter representation) by

$$
\begin{gathered}
\lambda(r)=e^{-\left(\sqrt{\left(1+r^{2}\right) / 2}-1\right)(1-x)}, \\
\varphi(r)=\left(1+\frac{r^{4}-1}{16}\right)^{-p} e^{-\left(\sqrt{\left(1+r^{2}\right) / 2}-1\right) x}, \quad 1 \leq r<\infty .
\end{gathered}
$$


In fact, let $r=\sqrt[4]{1+16 \xi^{2}}, \xi \in \mathbb{R}$, then $r \geq 1, \xi^{2}=\left(r^{4}-1\right) / 16$ and from (1.12) we know

$$
\cos \frac{\beta}{2}=\frac{\sqrt{1+r^{2}}}{\sqrt{2 r}} .
$$

Due to (3.8) there holds

$$
\widehat{A}^{*}(x) \widehat{A}(x)=e^{-\left(\sqrt{\left(1+r^{2}\right) / 2}-1\right)(1-x)},
$$

that is,

$$
\lambda(r)=e^{-\left(\sqrt{\left(1+r^{2}\right) / 2}-1\right)(1-x)} .
$$

Note that from (1.14), we know that the condition in (3.9),

$$
\left\|\left(1+\xi^{2}\right)^{p / 2} \hat{f}(\xi)\right\| \leq E
$$

is equivalent to

$$
\left\|\left(1+\xi^{2}\right)^{p / 2} e^{x \theta(\xi)} \widehat{u}(x, \xi)\right\| \leq E .
$$

Comparing this with (3.10), it is easy to see that

$$
\begin{aligned}
\varphi\left(\hat{A}^{*}(x) \hat{A}(x)\right) & =\left(1+\xi^{2}\right)^{-p} e^{-x(\theta(\xi)+\bar{\theta}(\xi))} \\
& =\left(1+\xi^{2}\right)^{-p} e^{-x\left(\sqrt[4]{1+16 \xi^{2}} \cos (\beta / 2)-1\right)} \\
& =\left(1+\frac{r^{4}-1}{16}\right)^{-p} e^{-\left(\sqrt{\left(1+r^{2}\right) / 2}-1\right) x}
\end{aligned}
$$

that is, $\varphi(r)=\left(1+\left(r^{4}-1\right) / 16\right)^{-p} e^{-\left(\sqrt{\left(1+r^{2}\right) / 2}-1\right) x}$.

Note 3.1. Here and following, we always denote by $\lambda$ the independent variable and $r$ the parameter.

In [12], we have proved the following propositions and obtained the best possible worst-case error $\omega(\delta, x)$ for problem (1.2).

Proposition 3.2 [12]. The function $\varphi(\lambda)$ defined by (3.11) is continuous and has the following properties:

(i) $\lim _{\lambda \rightarrow 0} \varphi(\lambda)=0$,

(ii) $\varphi(\lambda)$ is strictly monotonically increasing,

(iii) $\rho(\lambda):=\lambda \varphi^{-1}(\lambda)$ is strictly monotonically increasing and admits the parameter representation

$$
\begin{aligned}
& \lambda(r)=\left(1+\frac{r^{4}-1}{16}\right)^{-p} e^{-\left(\sqrt{\left(1+r^{2}\right) / 2}-1\right) x}, \\
& \rho(r)=\left(1+\frac{r^{4}-1}{16}\right)^{-p} e^{-\left(\sqrt{\left(1+r^{2}\right) / 2}-1\right)}, \quad 1 \leq r<\infty,
\end{aligned}
$$


(iv) $\rho^{-1}(\lambda)$ is strictly monotonically increasing and admits the parameter representation

$$
\begin{aligned}
\lambda(r) & =\left(1+\frac{r^{4}-1}{16}\right)^{-p} e^{-\left(\sqrt{\left(1+r^{2}\right) / 2}-1\right)}, \\
\rho^{-1}(r) & =\left(1+\frac{r^{4}-1}{16}\right)^{-p} e^{-\left(\sqrt{\left(1+r^{2}\right) / 2}-1\right) x}, \quad 1 \leq r<\infty,
\end{aligned}
$$

(v) for the inverse function $\rho^{-1}(\lambda)$ of $\rho(\lambda)$, there holds

$$
\rho^{-1}(\lambda)=\lambda^{x}\left[\frac{1}{\sqrt{2}} \ln \frac{1}{\lambda}\right]^{-4 p(1-x)}(1+o(1)) \quad \text { for } \lambda \longrightarrow 0 .
$$

Proposition 3.3 [12]. The function $\rho(\lambda)$ defined by (3.18) is strictly convex if and only if the following inequality holds:

$$
\left(p \Psi(r)+\frac{1}{\sqrt{2}} \Phi(r)\right)\left(p \Psi(r)+\frac{x}{\sqrt{2}} \Phi(r)\right) \Phi(r)+p(\dot{\Psi}(r) \Phi(r)-\Psi(r) \dot{\Phi}(r))>0, \quad r \geq 1,
$$

where

$$
\Psi(r):=\frac{4 r^{3}}{r^{4}+15}, \quad \Phi(r):=\frac{r}{\sqrt{1+r^{2}}}, \quad r \geq 1
$$

In particular, the function $\rho(\lambda)$ defined by (3.18) is strictly convex if $p=0, x>0$ or $p>0$, $x \geq 0$.

Due to Theorem 2.3, Propositions 3.2 and 3.3 we have proved in [12] the following optimal error bounds for problem (1.2), that is, the best possible worst-case error $\omega(\delta, x)$ defined by (3.2) for identifying the solution $u(x, t)$ of the problem (1.2) from noisy data $u^{\delta}(1, t)=g_{\delta}(t) \in L^{2}(\mathbb{R})$ under the condition (1.7) and $u(x, t) \in M_{p, E}$ given by (3.1).

Theorem 3.4 [12]. Let $\delta^{2} / E^{2} \leq 1$, then the following stability results hold:

(i) in case $p=0$ and $0<x<1$, there holds

$$
\omega(\delta, x)=E^{1-x} \delta^{x}
$$

(Hölder stability),

(ii) in case $p>0$ and $0 \leq x<1$, there holds

$$
\omega(\delta, x)=E^{1-x} \delta^{x}\left(\sqrt{2} \ln \frac{E}{\delta}\right)^{-2 p(1-x)}(1+o(1)) \quad \text { for } \delta \longrightarrow 0
$$

Now we consider the method of generalized Tikhonov regularization, apply it to problem (1.2), and show how to choose the regularization parameter such that it guarantees 
the optimal error bounds given by (3.23) and (3.24). This optimality result will be obtained by applying Theorem 2.4 to our transformed problem (3.5), it yields an optimal regularized approximation $\widehat{u}_{\alpha}^{\delta}(x, \xi)$ in the frequency domain. Due to Parseval formula

$$
\left\|\hat{u}_{\alpha}^{\delta}(x, \cdot)-\widehat{u}(x, \cdot)\right\|=\left\|u_{\alpha}^{\delta}(x, \cdot)-u(x, \cdot)\right\|,
$$

it follows that $u_{\alpha}^{\delta}(x, t)=\mathscr{F}^{-1}\left(\hat{u}_{\alpha}^{\delta}(x, \xi)\right)$ is optimal regularized approximations in the original domain.

The method of generalized Tikhonov regularization (2.7) applied to our problem (3.5) in the frequency domain consists in the determination of a regularized approximation $\widehat{u}_{\alpha}^{\delta}(x, \xi)$ by solving the minimization problem

$$
\begin{gathered}
\min _{\hat{v}(x, \cdot) \in L^{2}(\mathbb{R})} J_{\alpha}(\hat{v}(x, \cdot)), \\
J_{\alpha}(\widehat{v}(x, \cdot))=\left\|\hat{A}(x) \widehat{v}(x, \xi)-\hat{g}_{\delta}(\xi)\right\|^{2}+\alpha\left\|\left[\varphi\left(\hat{A}^{*}(x) \widehat{A}(x)\right)\right]^{-1 / 2} \widehat{v}(x, \xi)\right\|^{2} \\
=\left\|e^{-\theta(\xi)(1-x)} \hat{v}(x, \xi)-\hat{g}_{\delta}(\xi)\right\|^{2}+\alpha\left\|\left(1+\xi^{2}\right)^{p / 2} e^{(x / 2)(\theta(\xi)+\bar{\theta}(\xi))} \hat{v}(x, \xi)\right\|^{2},
\end{gathered}
$$

where $\theta(\xi)$ is given by (1.10), and

$$
\varphi\left(\widehat{A}^{*}(x) \hat{A}(x)\right)=\left(1+\xi^{2}\right)^{-p} e^{-x\left(\sqrt[4]{1+16 \xi^{2}} \cos (\beta / 2)-1\right)}
$$

Hence, $\hat{u}_{\alpha}^{\delta}(x, \xi)$ is the solution of Euler equation (comparing with (2.8)):

$$
\left(e^{-(\theta(\xi)+\bar{\theta}(\xi))(1-x)}+\alpha\left(1+\xi^{2}\right) p e^{(\theta(\xi)+\bar{\theta}(\xi)) x}\right) \widehat{u}_{\alpha}^{\delta}(x, \xi)=e^{-\bar{\theta}(\xi)(1-x)} \widehat{g}_{\delta}(\xi) .
$$

From (3.28) we conclude that the Tikhonov regularized solution $\hat{u}_{\alpha}^{\delta}(x, \xi)$ can be written in the form

$$
\begin{aligned}
\hat{u}_{\alpha}^{\delta}(x, \xi) & =\frac{e^{-\bar{\theta}(\xi)(1-x)}}{e^{-(\theta(\xi)+\bar{\theta}(\xi))(1-x)}+\alpha\left(1+\xi^{2}\right)^{p} e^{(\theta(\xi)+\bar{\theta}(\xi)) x}} \widehat{g}_{\delta}(\xi) \\
& =\frac{e^{\theta(\xi)(1-x)}}{1+\alpha\left(1+\xi^{2}\right)^{p} e^{(\theta(\xi)+\bar{\theta}(\xi))}} \hat{g}_{\delta}(\xi) .
\end{aligned}
$$

The following theorem is the main result of this paper and will answer the question how to choose the regularization parameter $\alpha=\alpha(x, \delta)$ in (3.29), such that the Tikhonov regularized solution $u_{\alpha}^{\delta}(x, t)=\mathscr{F}^{-1}\left(\hat{u}_{\alpha}^{\delta}(x, \xi)\right)$ is optimal on the set $M_{p, E}$ given by (3.1).

Theorem 3.5. Let $p=0, x>0$ or $p>0, x \geq 0$, and $\delta^{2} / E^{2} \leq 1$ hold, then the Tikhonov regularized solution $u_{\alpha}^{\delta}(x, t)=\mathscr{F}^{-1}\left(\hat{u}_{\alpha}^{\delta}(x, \xi)\right)$ with $\hat{u}_{\alpha}^{\delta}(x, \xi)$ given by (3.29) is optimal on 
the set $M_{p, E}$ provided the regularization parameter $\alpha$ is chosen optimally by

$$
\alpha_{0}=\frac{(1-x)\left(r_{0}^{4}+15\right)}{x\left(r_{0}^{4}+15\right)+4 \sqrt{2} p r_{0}^{2} \sqrt{1+r_{0}^{2}}}\left(\frac{\delta}{E}\right)^{2},
$$

where $r_{0}$ is the (unique) solution of the equation

$$
\lambda(r) \varphi(r)=\left(1+\frac{r^{4}-1}{16}\right)^{-p} e^{-\left(\sqrt{\left(1+r^{2}\right) / 2}-1\right)}=\left(\frac{\delta}{E}\right)^{2} .
$$

In the case $x=0$ and $p>0$ there holds

$$
\alpha_{0}=\frac{1}{2 p}\left(\ln \frac{E}{\delta}\right)\left(\frac{\delta}{E}\right)^{2}(1+o(1)) \quad \text { for } \delta \longrightarrow 0
$$

and in the case $0<x<1, p \geq 0$, there holds

$$
\alpha_{0}=\frac{1-x}{x}\left(\frac{\delta}{E}\right)^{2}(1+o(1)) \quad \text { for } \delta \longrightarrow 0
$$

Furthermore, the optimal error estimate $\left\|u_{\alpha_{0}}^{\delta}(x, t)-u(x, t)\right\| \leq \omega(\delta, x)$ holds true where $\omega(\delta, x)$ is given by (3.23) and (3.24), respectively.

Proof. From Theorem 2.4 it follows that the optimal regularization parameter $\alpha$ is given by $(2.10)$ with $\varphi(\lambda)$ given by (3.11), which is equivalent to

$$
\alpha_{0}=\frac{\varphi\left(\lambda_{0}\right)}{\lambda_{0} \varphi^{\prime}\left(\lambda_{0}\right)}\left(\frac{\delta}{E}\right)^{2} \quad \text { with } \varphi\left(\lambda_{0}\right)=\rho^{-1}\left(\frac{\delta^{2}}{E^{2}}\right) .
$$

Note that $\varphi\left(\lambda_{0}\right)=\rho^{-1}\left((\delta / E)^{2}\right)$ can be rewritten as $\rho\left(\varphi\left(\lambda_{0}\right)\right)=(\delta / E)^{2}$, that is,

$$
\varphi\left(\lambda_{0}\right) \lambda_{0}=\left(\frac{\delta}{E}\right)^{2}
$$

Its parameter representation is just (3.31), so $r_{0}$ should be the solution of (3.31). Moreover, because of the strict monotonicity of functions $\lambda(r)$ and $\varphi(r), r_{0}$ should be the unique solution of (3.31).

Note that from (3.11), we know that

$$
\begin{gathered}
\dot{\varphi}(r)=-\left(\frac{p\left(r^{3} / 4\right)}{1+\left(r^{4}-1\right) / 16}+\frac{x}{\sqrt{2}} \frac{r}{\sqrt{1+r^{2}}}\right) \varphi(r), \\
\dot{\lambda}(r)=-\frac{1-x}{\sqrt{2}} \frac{r}{\sqrt{1+r^{2}}} \lambda(r) .
\end{gathered}
$$


So,

$$
\varphi^{\prime}(\lambda)=\frac{\dot{\varphi}(r)}{\dot{\lambda}(r)}=\frac{\left(p\left(r^{3} / 4\right) /\left(1+\left(r^{4}-1\right) / 16\right)+(x / \sqrt{2})\left(r / \sqrt{1+r^{2}}\right)\right) \varphi(r)}{((1-x) / \sqrt{2})\left(r / \sqrt{1+r^{2}}\right) \lambda(r)},
$$

and $\alpha_{0}$ in (3.34) can be rewritten as

$$
\alpha_{0}=\frac{\varphi\left(r_{0}\right)}{\lambda\left(r_{0}\right)\left(\dot{\varphi}\left(r_{0}\right) / \dot{\lambda}\left(r_{0}\right)\right)}\left(\frac{\delta}{E}\right)^{2}=\frac{(1-x)\left(r_{0}^{4}+15\right)}{x\left(r_{0}^{4}+15\right)+4 \sqrt{2} p r_{0}^{2}}\left(\frac{\delta}{E}\right)^{2},
$$

where $r_{0}$ is the solution of (3.31), this is just the representation formula (3.30). In addition, due to (3.31), we have

$$
-p \ln \left(1+\frac{r_{0}^{4}-1}{16}\right)-\left(\sqrt{\frac{1+r_{0}^{2}}{2}}-1\right)=2 \ln \frac{\delta}{E}
$$

Note that for $r_{0} \rightarrow+\infty$ when $\delta \rightarrow 0$, there holds

$$
\frac{r_{0}}{\sqrt{2}}(1+o(1))=2 \ln \frac{E}{\delta} \quad \text { for } \delta \longrightarrow 0
$$

that is,

$$
r_{0}=2 \sqrt{2} \ln \frac{E}{\delta}(1+o(1)) \quad \text { for } \delta \longrightarrow 0
$$

The asymptotical representation of $\alpha_{0}$ can be given as follows.

(i) $x=0, p>0$. From (3.30), (3.41), and note that $r_{0} \rightarrow \infty$ for $\delta \rightarrow 0$, we have

$$
\begin{aligned}
\alpha_{0} & =\frac{r_{0}^{4}+15}{4 \sqrt{2} p r_{0}^{2} \sqrt{1+r_{0}^{2}}}\left(\frac{\delta}{E}\right)^{2} \\
& =\frac{r_{0}^{4}\left(1+\left(15 / r_{0}^{4}\right)\right)}{4 \sqrt{2} p r_{0}^{3} \sqrt{1+1 / r_{0}^{2}}}\left(\frac{\delta}{E}\right)^{2} \\
& =\frac{r_{0}}{4 \sqrt{2} p}\left(\frac{\delta}{E}\right)^{2}(1+o(1)) \text { for } \delta \longrightarrow 0 \\
& =\frac{1}{4 \sqrt{2} p} 2 \sqrt{2}\left(\ln \frac{E}{\delta}\right)\left(\frac{\delta}{E}\right)^{2}(1+o(1)) \text { for } \delta \longrightarrow 0 \\
& =\frac{1}{2 p}\left(\ln \frac{E}{\delta}\right)\left(\frac{\delta}{E}\right)^{2}(1+o(1)) \quad \text { for } \delta \longrightarrow 0 .
\end{aligned}
$$

This is just the formula (3.32). 
(ii) $0<x<1, p \geq 0$. In this case, from (3.30) we have

$$
\begin{aligned}
\alpha_{0} & =\frac{r_{0}^{4}(1-x)\left(1+15 / r_{0}^{4}\right)}{r_{0}^{4}\left(x\left(1+15 / r_{0}^{4}\right)+4 \sqrt{2} p\left(\sqrt{1+r_{0}^{2}} / r_{0}^{2}\right)\right)}\left(\frac{\delta}{E}\right)^{2} \\
& =\frac{1-x}{x}\left(\frac{\delta}{E}\right)^{2}(1+o(1)) \quad \text { for } \delta \longrightarrow 0 .
\end{aligned}
$$

This is just the formula (3.33). It is the conclusion of Theorem 2.4 that the optimal error estimates (3.23) and (3.24) hold for $\alpha_{0}$ given by (3.32) and (3.33), respectively.

The proof of Theorem 3.5 is complete.

\section{A numerical example}

It is easy to verify that the function

$$
u(x, t)= \begin{cases}\frac{x+1}{t^{3 / 2}} \exp \left\{-\frac{(x+1-t)^{2}}{4 t}\right\}, & t>0,0 \leq x<1 \\ 0, & t \leq 0\end{cases}
$$

is the exact solution of problem (1.2) with data

$$
\begin{gathered}
g(t)= \begin{cases}\frac{2}{t^{3 / 2}} \exp \left\{-\frac{(2-t)^{2}}{4 t}\right\}, & t>0, \quad g(t) \in L^{2}(\mathbb{R}), \\
0, & t \leq 0,\end{cases} \\
f(t):=u(0, t)=\left\{\begin{array}{ll}
\frac{1}{t^{3 / 2}} \exp \left\{-\frac{(1-t)^{2}}{4 t}\right\}, & t>0, \\
0, & t \leq 0,
\end{array} \quad f(t) \in L^{2}(\mathbb{R}) .\right.
\end{gathered}
$$

Figures 4.1, 4.2, and 4.3 give the comparison of the approximation solution with exact solution at $x=0,0.1$, and 0.9 , respectively. The tests were performed in the following way: first, we add a normally distributed perturbation of variance $10^{-4}$ to each function, giving vectors $\left\{g_{\delta}^{(m)}\right\}_{m=1}^{100}$, then we use the formula

$$
u_{\alpha}^{\delta}(x, t)=\frac{1}{\sqrt{2 \pi}} \int_{-\infty}^{\infty} e^{i \xi t} \frac{e^{\theta(\xi)(1-x)}}{1+\alpha\left(1+\xi^{2}\right)^{p} e^{(\theta(\xi)+\bar{\theta}(\xi))}} \widehat{g}_{\delta}(\xi) d \xi
$$

to compute the Tikhonov approximation solution, the data $\delta$ is given according to (1.7).

It can be seen from these figures that the computational effect of the optimal Tikhonov regularization method is fairly satisfactory.

Remark 4.1. In our numerical experiment, if $E$ is considered as a function of $p$, we can find the relation between $E(p)$ and $p$ in Table 4.1. From the latter, we conclude that the function $f(\cdot)$ belongs to $H^{p}(\mathbb{R})$, where $p$ satisfies $0 \leq p \leq 2$. The function $E(p)$ is plotted as in Figure 4.4. 


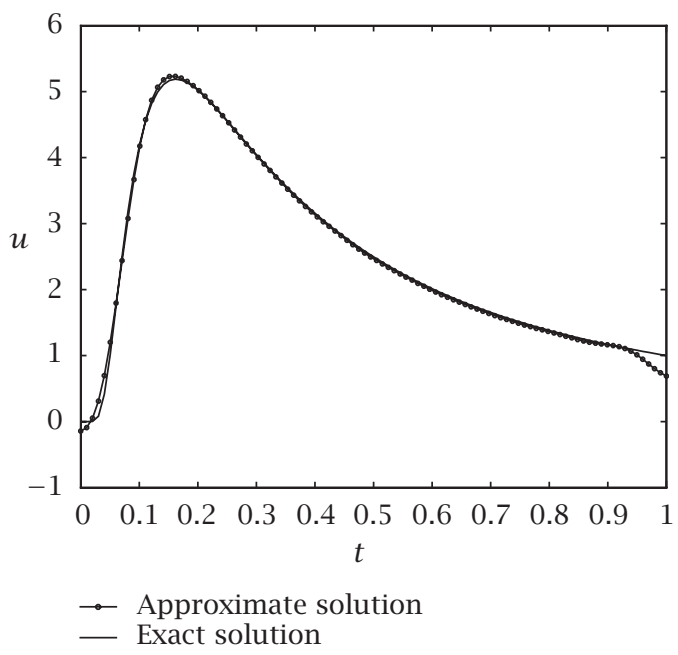

Figure 4.1. $x=0, p=1 / 3, \delta \doteq 0.002, E=\|f\|_{1 / 3} \doteq 4.39, \alpha_{0} \doteq 2.5 \times 10^{-6}$ (by formula (3.32)).

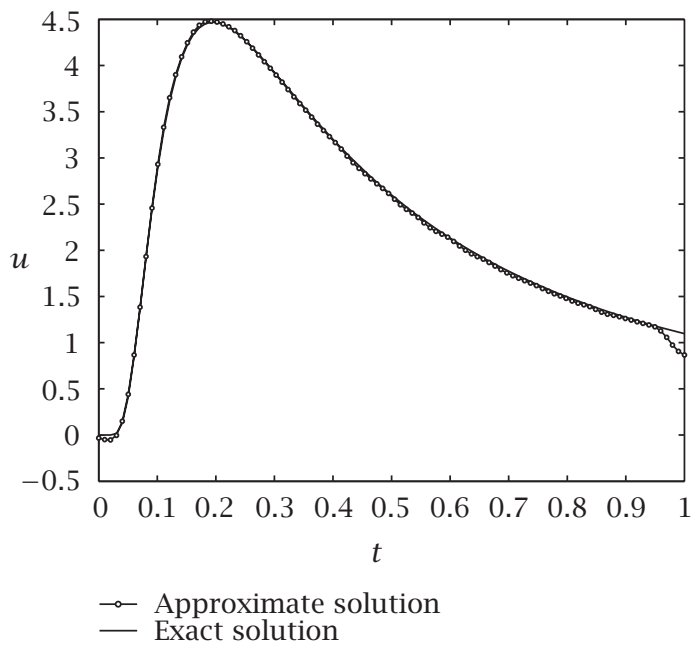

Figure 4.2. $x=0.1, p=0, \delta \doteq 0.002, E=\|f\| \doteq 3, \alpha_{0} \doteq 4.0 \times 10^{-6}$ (by formula (3.33)).

For fixed $\delta$ and $x$, we take $\delta=0.1$ and $x=0$, then the best possible worst error (3.24) becomes

$$
\omega(p, 0.1,0)=E(p)\left(\sqrt{2} \ln \left(\frac{E(p)}{0.1}\right)\right)^{-2 p} \quad \text { for } 0 \leq p \leq 2 .
$$

We can plot the figure of $\omega(p, 0.1,0)=: \omega(p)$ as in Figure 4.5. 


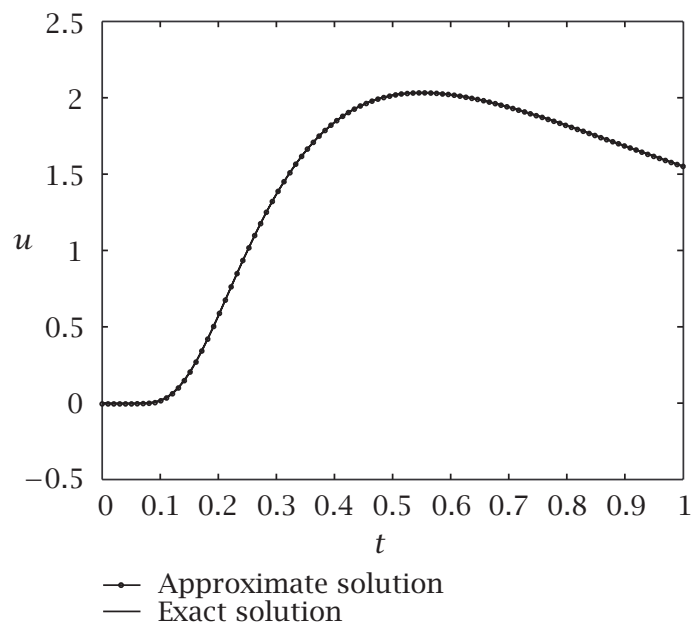

Figure 4.3. $x=0.9, p=0, \delta \doteq 0.002, E=\|f\| \doteq 3, \alpha_{0} \doteq 4.0 \times 10^{-8}$ (by formula (3.33)).

Table 4.1

\begin{tabular}{cccccccccccc}
\hline$p$ & 0 & 0.1 & 0.2 & 0.3 & 0.4 & 0.5 & 0.6 & 0.7 & 0.8 & 0.9 & 1.0 \\
\hline$E(p)$ & 2.97 & 3.28 & 3.67 & 4.18 & 4.86 & 5.75 & 6.95 & 8.57 & 10.75 & 13.75 & 17.86 \\
\hline
\end{tabular}

\begin{tabular}{ccccccccccc}
\hline$p$ & 1.1 & 1.2 & 1.3 & 1.4 & 1.5 & 1.6 & 1.7 & 1.8 & 1.9 & 2.0 \\
\hline$E(p)$ & 23.57 & 31.54 & 42.79 & 58.75 & 81.59 & 114.55 & 162.47 & 232.68 & 336.30 & 490.38 \\
\hline
\end{tabular}

\begin{tabular}{cccccc}
\hline$p$ & 3 & 4 & 5 & 6 & 7 \\
\hline$E(p)$ & $3.2225 * 10^{4}$ & $3.85886 * 10^{6}$ & $7.32224 * 10^{8}$ & $2.01914 * 10^{11}$ & $7.62738 * 10^{13}$ \\
\hline
\end{tabular}

In this experiment, we can conclude that $\omega(p)$ is a decreasing function in respect of $p$. The same result can be found when the various data $\delta$ is given. In theory, the monotony of the function $\omega(p, \omega, x)=\delta^{x}\left[E(p)(\sqrt{2} \ln E(p) / \delta)^{-2 p}\right]^{1-x}$ depends on $\delta$ and $E(p)$.

We define 2-norm of a vector $V=\left(v_{i}\right)_{i=1}^{m}$,

$$
|V|_{2}:=\sqrt{\frac{1}{m} \sum_{i=1}^{m} v_{i}}
$$

Hence we can compute the error between the exact solution and approximation

$$
\Delta_{e}=\left|\left(u\left(0, t_{i}\right)\right)_{i=1}^{m}-\left(u_{\alpha}^{\delta}\left(0, t_{i}\right)\right)_{i=1}^{m}\right|_{2} .
$$


Chu-Li Fu et al. 1235

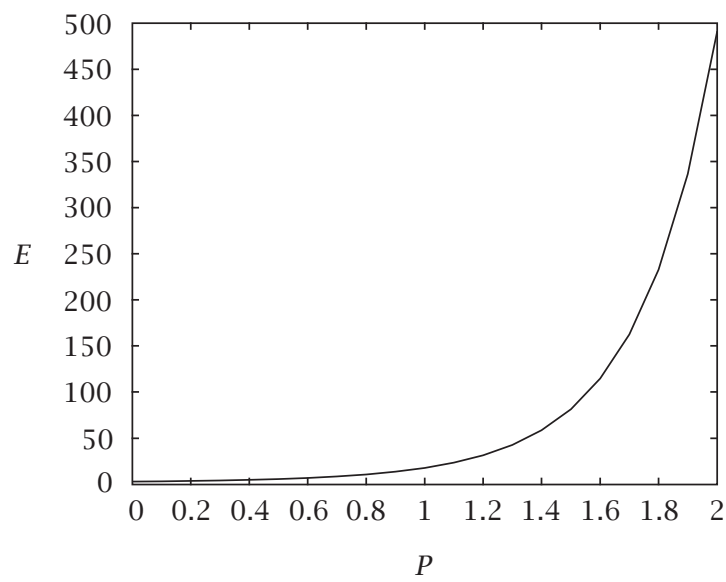

Figure 4.4. $E(p)$.

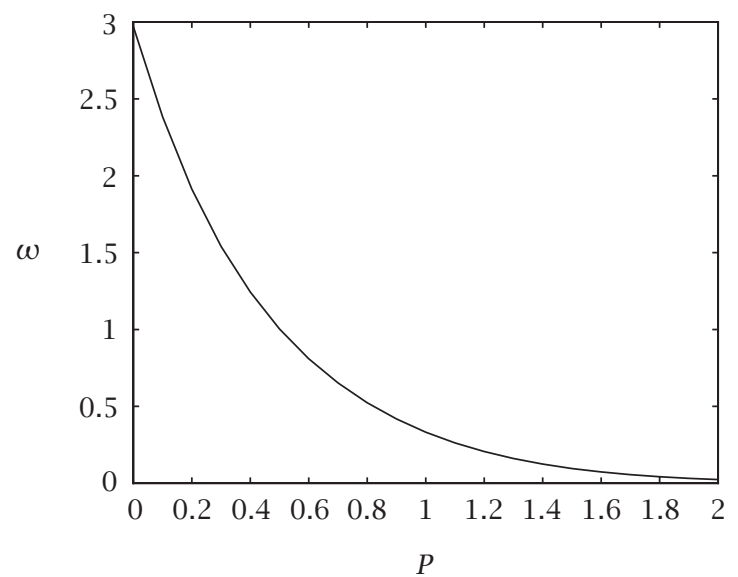

Figure 4.5. $\omega(p)$.

Now we use noise data $\left(g_{\delta}\right)_{i}=(g)_{i}+\epsilon$, where $(g)_{i}$ is the discretized exact data. Hence

$$
\delta:=\sqrt{\frac{1}{m} \sum_{i=1}^{m}\left(\left(g_{\delta}\right)_{i}-(g)_{i}\right)}=\sqrt{\frac{1}{m} \sum_{i=1}^{m} \epsilon}=\epsilon .
$$

The results of fixing $\delta=\epsilon=0.10, x=0$ are presented in Table 4.2, which shows that our theoretical optimal error estimate is decreasing with the index $p$. 
Table 4.2

\begin{tabular}{cccccccc}
\hline$p$ & 0.1 & 0.3 & 0.5 & 0.8 & 1.0 & 1.5 & 2.0 \\
\hline$\Delta_{e}(p)$ & 0.6257 & 0.5244 & 0.5175 & 0.5142 & 0.5046 & 0.4576 & 0.3994 \\
\hline
\end{tabular}

\section{Acknowledgment}

The project is supported by the National Natural Science Foundation of China (no. 10271050) and the Natural Science Foundation of Gansu Province of China (no. ZS021A25-001-Z).

\section{References}

[1] J. V. Beck, Nonlinear estimation applied to the nonlinear inverse heat conduction problem, Int. J. Heat Mass Transfer 13 (1970), 703-716.

[2] J. V. Beck, B. Blackwell, and S. R. Clair, Inverse Heat Conduction: Ill-posed Problems, John Wiley and Sons, New York, 1985.

[3] J. R. Cannon, The One-Dimensional Heat Equation, Encyclopedia of Mathematics and its Applications, vol. 23, Addison-Wesley Publishing Company Advanced Book Program, Massachusetts, 1984.

[4] A. Carasso, Determining surface temperatures from interior observations, SIAM J. Appl. Math. 42 (1982), no. 3, 558-574.

[5] J. Cheng and M. Yamamoto, One new strategy for a priori choice of regularizing parameters in Tikhonov's regularization, Inverse Problems 16 (2000), no. 4, L31-L38.

[6] L. Eldén, Numerical solution of the sideways heat equation by difference approximation in time, Inverse Problems 11 (1995), no. 4, 913-923.

[7] _ Solving an inverse heat conduction problem by "a method of lines," J. Heat Transfer, Trans. ASME 119 (1997), 406-412.

[8] H. W. Engl, M. Hanke, and A. Neubauer, Regularization of Inverse Problems, Mathematics and its Applications, vol. 375, Kluwer Academic Publishers, Dordrecht, 1996.

[9] C. L. Fu, Simplified Tikhonov and Fourier regularization methods on a general sideways parabolic equation, J. Comput. Appl. Math. 167 (2004), no. 2, 449-463.

[10] C. L. Fu and C. Y. Qiu, Wavelet and error estimation of surface heat flux, J. Comput. Appl. Math. 150 (2003), no. 1, 143-155.

[11] C. L. Fu, C. Y. Qiu, and Y. B. Zhu, Wavelet regularization with error estimates on a general sideways parabolic equation, Appl. Math. Lett. 16 (2003), no. 4, 475-479.

[12] C. L. Fu, X. T. Xiong, H. F. Li, and Y. B. Zhu, Wavelet and spectral regularization methods for a sideways parabolic equation, Appl. Math. Comput. 160 (2005), no. 3, 881-908.

[13] C. L. Fu, Y. B. Zhu, and C. Y. Qiu, Wavelet regularization for an inverse heat conduction problem, J. Math. Anal. Appl. 288 (2003), no. 1, 212-222.

[14] D. N. Hào and H.-J. Reinhardt, On a sideways parabolic equation, Inverse Problems 13 (1997), no. 2, 297-309.

[15] F. Natterer, Error bounds for Tikhonov regularization in Hilbert scales, Applicable Anal. 18 (1984), no. 1-2, 29-37.

[16] C. Y. Qiu and C. L. Fu, A note on: "Sideways heat equation and wavelets" and constant $e^{*}$, Comput. Math. Appl. 43 (2002), no. 8-9, 1125-1134.

[17] Wavelet regularization for an ill-posed problem of the parabolic equation, Acta Math. Sci. Ser. A Chin. Ed. 22 (2002), no. 3, 361-372.

[18] C. Y. Qiu, C. L. Fu, and Y. B. Zhu, Wavelets and regularization of the sideways heat equation, Comput. Math. Appl. 46 (2003), no. 5-6, 821-829. 
[19] T. Regińska, Sideways heat equation and wavelets, J. Comput. Appl. Math. 63 (1995), no. 1-3, 209-214.

[20] T. I. Seidman and L. Eldén, An "optimal filtering" method for the sideways heat equation, Inverse Problems 6 (1990), no. 4, 681-696.

[21] U. Tautenhahn, Optimal stable approximations for the sideways heat equation, J. Inverse Ill-Posed Probl. 5 (1997), no. 3, 287-307.

[22] Optimality for ill-posed problems under general source conditions, Numer. Funct. Anal. Optim. 19 (1998), no. 3-4, 377-398.

[23] X.-T. Xiong, C. L. Fu, and H.-F. Li, Central difference schemes in time and error estimate on a non-standard inverse heat conduction problem, Appl. Math. Comput. 157 (2004), no. 1, 77-91.

Chu-Li Fu: Department of Mathematics, Lanzhou University, Lanzhou 730000, China E-mail address: fuchuli@lzu.edu.cn

Hong-Fang Li: Department of Mathematics, Lanzhou University, Lanzhou 730000, China E-mail address: lihf04@st.lzu.edu.cn

Xiang-Tuan Xiong: Department of Mathematics, Lanzhou University, Lanzhou 730000, China E-mail address: xiongxt04@st.lzu.edu.cn

Peng Fu: Institute of Network, School of Electronics and Information Engineering, Xi'an Jiaotong University, Xi'an 710049, China

E-mail address: fupeng@xanet.edu.cn 


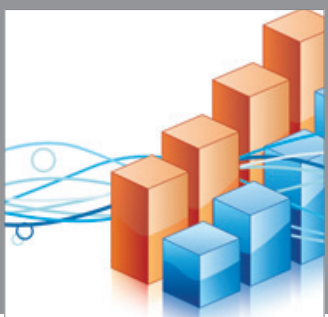

Advances in

Operations Research

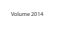

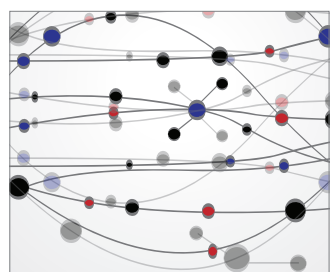

\section{The Scientific} World Journal
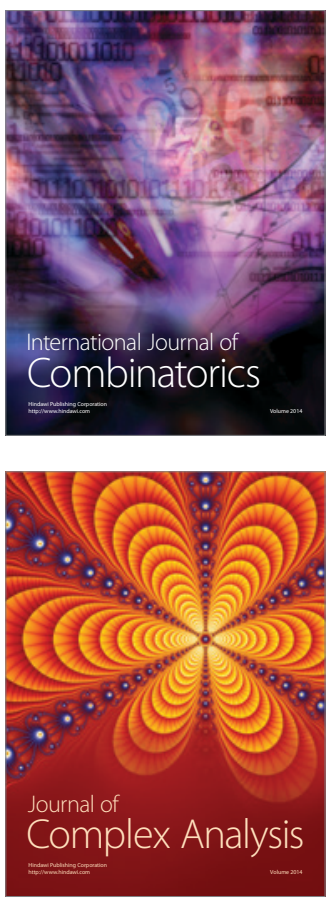

International Journal of

Mathematics and

Mathematical

Sciences
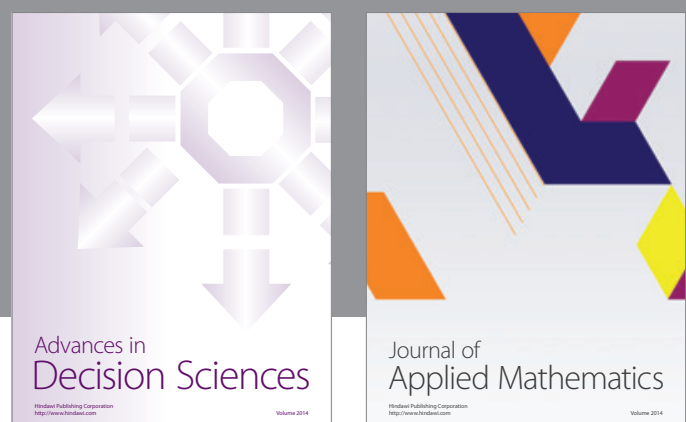

Journal of

Applied Mathematics
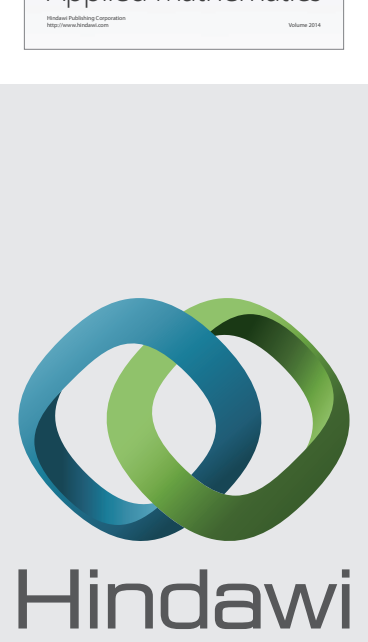

Submit your manuscripts at http://www.hindawi.com
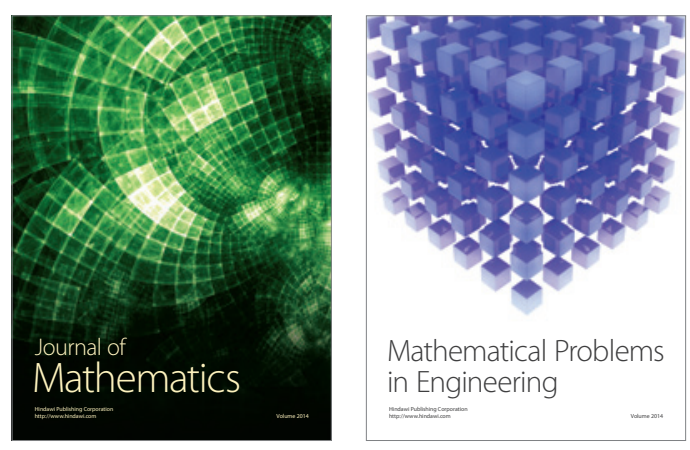

Mathematical Problems in Engineering
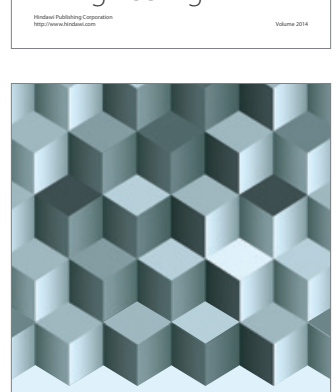

Journal of

Function Spaces
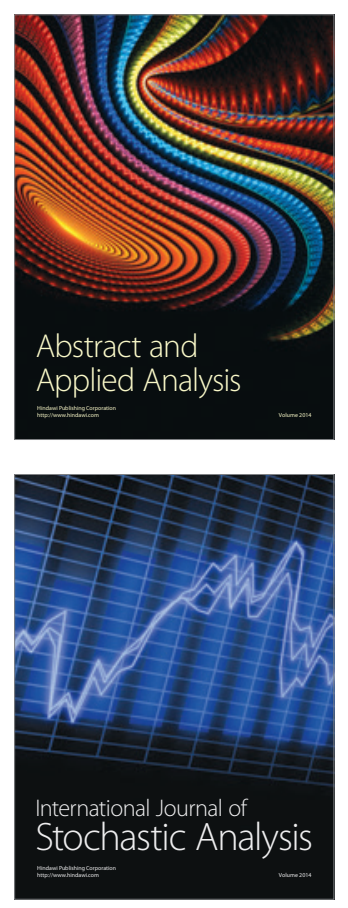

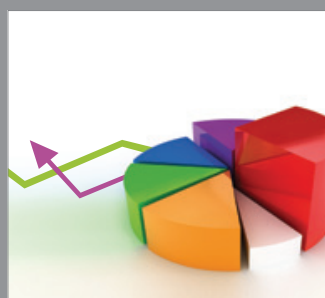

ournal of

Probability and Statistics

Promensencen
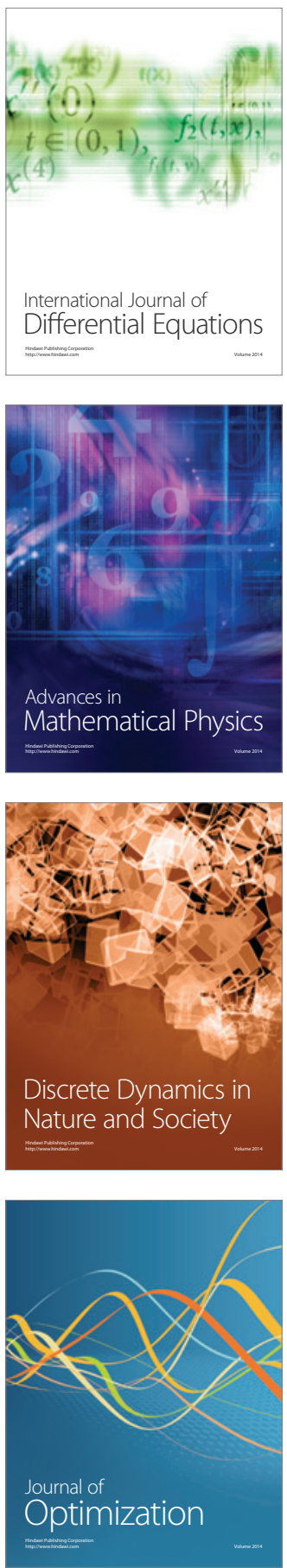